\title{
EL JUEGO COMO RECURSO DIDÁCTICO PARA LA ENSEÑANZA DE LAS CIENCIAS: MATEMATICAS Y QUIMICA
}

\author{
PLAY AS AN INSTRUCTIONAL MATERIAL IN SCIENCE
} TEACHING: MATHEMATICS AND CHEMISTRY

\author{
Mayra Adilene López Vázquez ${ }^{1}$ \\ adilene.lopez5a@gmail.com \\ Verónica García Martínez ${ }^{2}$ \\ veronica.garcia@ujat.mx
}

1 Consejo Nacional de Fomento Educativa, México

2 Universidad Juárez Autónoma de Tabasco, México 
Para citar este artículo:

López V. Mayra, García Verónica. (2020). El juego como recurso didáctico para la enseñanza

de las ciencias: matemáticas y química. Espacio I+D, Innovación más Desarrollo. IX(23),

39-53. doi: http://dx.doi.org/10.31644/IMASD.23.2020.a03

\section{RESUMEN}

El presente trabajo tiene como propósito presentar al juego como un recurso que se ha convertido en una herramienta de apoyo en la enseñanza de las ciencias, a partir de una investigación documental. Se exponen sus ventajas, sus características y tipologías. Posteriormente se ofrecen, en tablas sintéticas, ejemplos de propuestas que se implementaron y documentaron para mostrar las potencialidades de algunos juegos en la enseñanza de Matemáticas y Química, en varios niveles educativos. Se concluye que, en la didáctica de las ciencias, el juego representa un recurso importante, aunque muy poco valorado por el profesorado, pero necesario para la formación del mismo.

\section{Palabras clave}

Juego; recurso didáctico; enseñanza de ciencias. 


\section{- Abstract-}

The purpose of this work is to present the play as an aid in the teaching of science, from a documentary investigation. Game typologies, characteristics and advantages are presented. Then, a chart is shown with examples of proposals implemented and documented to show the potential of some games in the teaching of mathematics and chemistry, in some levels of education. It is concluded that in the teaching of sciences, play is an important aid, even necessary, although undervalued by teachers.

\section{Keywords}

Game, instructional material, science teaching. 
$\mathrm{E}$ 1 juego está asociado con la diversión y el placer (Bernabeu y Goldstein, 2008; Chacón, 2008), aunque también se define como un "ejercicio recreativo o de competición sometido a reglas, y en el cual se gana o se pierde" (Sánchez, 2016, p.18). Sin embargo, el juego de acuerdo con algunos autores como Melo y Hernández (2014), no se reduce a una simple actividad libre y espontánea, sino que se encuentra supeditada a reglas que el sujeto acepta obedecer de manera voluntaria, y lejos de que éstas sean motivo de desagrado las integra como parte del ejercicio, e incluso, las disfruta. De modo que el juego no sólo representa una forma de goce o pasatiempo, sino que cumple con una función esencial en el desarrollo del individuo, ya que le permite descubrir y explorar el mundo que le rodea, así como establecer relaciones con su entorno y con sus semejantes.

En este sentido Gallardo (2018) estima que las funciones de aprendizaje y socialización están intrínsecas en el juego, su papel es fundamental en el progreso del ser humano.

En la historia de la humanidad, se colocó al juego como una forma de ocio, poco relacionada con el trabajo o el conocimiento, sin embargo, en los últimos años algunos autores Melo y Hernández (2014), Plutin y García (2016), Muñiz y Rodríguez (2014), lo han propuesto como una estrategia eficaz en el aprendizaje, principalmente de niños y jóvenes. Adicional al desarrollo intelectual y socio afectivo que pueda tener, el juego fortalece el desarrollo físico y psicomotor, al ser un hecho motriz implícito en la práctica cotidiana del niño (Molina, 2016 citado por Morales y Urrego, 2017).

En virtud de lo anterior, el objetivo de este trabajo es presentar al juego como una posibilidad o recurso didáctico de apoyo en la enseñanza. De acuerdo con la literatura, es utilizado en diversas disciplinas para estimular el aprendizaje, pero la mayor dificultad para los docentes de nivel básico y medio es la enseñanza de las ciencias, sobre todo en asignaturas como Matemáticas y Química. Solbes Lozano y García afirman que los estudiantes tienden a considerar las asignaturas de corte científico como las más aburridas, lo cual "puede provocar una desmotivación a los estudiantes, y es evidente que la motivación es uno de los pilares de la didáctica. Sin motivación no hay aprendizaje efectivo" (2008, p.65).

Por tal motivo, este trabajo se aproxima a las experiencias que se han desarrollado sobre todo en algunos países de Iberoamérica, en las que el juego ha servido para estimular a los estudiantes en este tipo de asignaturas, de modo que puedan servir como inspiración a otros docentes en su trabajo en el aula.

Para tal fin se utilizó la metodología de análisis de contenido, a través del buscador Google Académico, así como la consulta de la base de datos, EBSCO y Redalyc. Se introdujeron las palabras clave: juego, estrategia didáctica y ciencias. Se acopiaron publicaciones académicas que abordaban el tema y se seleccionaron aquellas que cumplieran los siguientes criterios: 
a) Que tuvieran una fecha no mayor a a 10 años.

b) Que se enfocaran al contexto de habla hispana en diferentes países.

c) Que relataran una experiencia del uso del juego en la enseñanza de las ciencias.

Además de seleccionar los artículos que hablaban de experiencias en particular, se tomaron en consideración muchos otros que aportaron conceptos de acuerdo con el propósito del presente trabajo.

\section{EL JUEGO COMO RECURSO DIDÁCTICO}

La efectividad del juego descansa en el hecho de que es una actividad inherente al ser humano, vinculada al gozo, al placer y a la diversión. Melo y Hernández (2014) afirman que forma parte de las manifestaciones humanas en todas las etapas de la vida, y que debe pensarse en él como un recurso que permite construir conocimiento. El juego es una actividad concebida inicialmente como de asueto o entretenimiento, en los últimos años se ha considerado como un recurso didáctico para las aulas escolares, en donde se le permite al estudiante jugar, con el propósito de favorecer su aprendizaje. Los docentes han descubierto sus beneficios en el espacio áulico. De acuerdo a la literatura consultada se pueden destacar los siguientes:

a) Es un activador de la conducta humana. Para algunos autores como Ruiz (2017), el juego representa algo más que una simple actividad humana, ya que lo catalogan como algo indispensable y vital para el desarrollo del individuo, no sólo para los niños, sino también para los mayores. Brousseau (1997, citado por González, Molina y Sánchez, 2014) sostiene que éste puede referirse a la ejecución de actividades físicas o mentales, cuyo objetivo esencial es proveer deleite. Jugar permite ensayar conductas que más tarde se extrapolan a lo social, y es útil para el desarrollo de habilidades motrices, cognitivas y afectivas (Pacheco, 2011).

b) Provee de gratificaciones y placer. Se concibe al juego como una actividad lúdica, de recreación, que proporciona gozo y alegría y que se puede practicar a cualquier edad (Melo y Hernández, 2014; Gallardo, 2018). El juego se sitúa como una actividad emotiva, que no se limita a una etapa de la vida del ser humano. Pacheco (2011) destaca que éste suele ser placentero, divertido y alegre. "Tiene una función potenciadora del desarrollo y el aprendizaje: a través del juego y debido a las características de éste, se convierte en un medio idóneo para el aprendizaje, ya que con él las personas encuentran una motivación para aprender...” (p.15). Un alumno más 
relajado, confiado, desinhibido y con grandes deseos de aprender para ganar, es el resultado del uso del juego en el aula, donde el estudiante se transforma y cambia su conducta haciéndose más proclive al aprendizaje (Castrillón, 2017).

c) Estimula la imaginación y la creatividad. Calderón (2013, citado por Montero, 2017), propone tres características del juego didáctico: espontáneo, motivador y estimulador de la imaginación. Es espontáneo porque en el caso de los niños surge sin planearlo, simplemente aprovechan cualquier momento o lugar para jugar de forma natural.

d) Es motivador, porque representa una actividad gratificante que genera un impulso intrínseco de satisfacción en quienes lo realizan. Además, estimula la imaginación en virtud de que, mientras se juega, se llevan a cabo procesos mentales que permiten vivenciar o actuar lo imaginario en un plano real.

e) Ayuda el en desarrollo de habilidades y competencias. Enmarcado en una actividad didáctica, el juego se reconoce como un potenciador del desarrollo cognitivo, afectivo y comunicativo, elementos que conforman la construcción social del conocimiento (Melo y Hernández, 2014). Además, la actividad lúdica representa uno de los mecanismos más eficaces para el aprendizaje de nuevas habilidades, destrezas, experiencias y conceptos (Domínguez, 2015). A través del juego se puede estimular una serie de competencias en los estudiantes, entre las que destacan las competencias comunicativas (Palacino, (2007)

Se han propuesto diversas clasificaciones del juego como recurso de enseñanza, la más aceptada es la creada por Piaget, quien estudió el desarrollo y aprendizaje del niño y las implicaciones que el juego tiene sobre él. De acuerdo con Piaget (citado por Cruz, 2013), existen: a) juegos prácticos, que consisten en la repetición de secuencias bien establecidas de acciones, sin propósito alguno. b) simbólicos, en los que el niño disfruta de imitar acciones de la vida diaria y c) de reglas, que es una forma de juego más colectivo y que está constituido por reglas establecidas o espontáneamente determinadas que se realizan con dos o más personas.

Otra clasificación es la presentada por Groos (1902, citado por Cruz, 2013), el cual tipificó los juegos en dos grandes grupos: a) los de experimentación o funciones generales, que incluyen los juegos sensoriales, motores e intelectuales y b) los afectivos o de ejercitación de la voluntad, los cuales son juegos de funciones especiales, que comprenden aquellos de persecución, de lucha, de ocultamiento, de caza, de imitación, de actividades familiares y sociales. 
Una propuesta más es la de Gairín (1990), quien clasificó los juegos en dos grupos: a) de conocimiento, mismos que se subcategorizan en preinstruccionales (familiarizan al alumno con un concepto), co-instruccionales (se suman a las actividades de enseñanza), post-instruccionales (útiles para consolidar el aprendizaje), y b) de estrategia, que pueden ser solitarios o multipersonales.

Otra clasificación es la de Aizencang (citado por Melo y Hernández, 2014), quien divide los juegos en los siguientes tipos: a) Agon o juegos de competencia, donde se establece una lucha de poder en la que se comparte un mismo espacio; b) Alea o juegos de suerte, en que se emplea la adivinación y la profecía; c) Mimesis o juegos de imitación, en los que se practican comúnmente las artes y el teatro. Ese tipo de juegos "representan la primera muestra de aprendizaje del niño, el cual imita profesiones u oficios que más adelante asumirá como verdaderos" (p. 51); d) Ilinx o juegos de vértigo, relacionados con actividades deportivas. Tener en cuenta las diversas clasificaciones existentes, resulta útil para saber qué tipo de juegos se pueden implementar de acuerdo con el objetivo que se quiera lograr, y las asignaturas que se desea apoyar.

Aunque el juego haya sido estudiado como dinamizador del aprendizaje, no se le ha conferido, como tal, ese valor: Sin embargo, ha emergido en los últimos años una metodología que considera al juego como una excelente herramienta para aumentar la concentración, el esfuerzo y la motivación, fundada en el reconocimiento, la competencia, la colaboración y otras potencialidades educativas (Sánchez y Francesc, 2015) que se denomina gamificación o ludificación. Esta metodología, que también es considerada técnica, método o estrategia (Hierro, 2014), es un "procedimiento que reubica a un sistema tradicional de aprendizaje de conocimientos, que tiene como objetivo obtener resultados satisfactorios por los estudiantes en el área educativa, a través de juegos netamente académicos, que sustentan el aprendizaje"(Rengel, 2018, p.3), todo esto en un contexto de no juego, es decir, cuando la diversión, no es el fin, sino el medio, ya que el fin en este concepto es el aprendizaje, especialemente colaborativo.

Esta tendencia surge en el ámbito empresarial y está asociada al uso de la tecnología para fines didácticos, en especial los videojuegos (Marín, 2015) y se utiliza más en el aprendizaje de adultos. Es empleada en los ambientes de negocios y universidades ya que incentiva la competencia con el afán de desarrollar la competitividad. De hecho, se cuestiona si gamificar una actividad de aprendizaje en el espacio aúlico la convierte en lúdica, pues es una actividad de aprendizaje más, sólo que con particularidades de diseño cuya finalidad pedagógica va más allá de motivar (Foncubierta y Rodríguez, 2014).

El inconveniente de este método es que requiere del conocimiento profundo de la metodología, pues se parte del objetivo de aprendizaje para 
desarrollar el juego, es decir no se puede improvisar la planeación para la participación activa de los jugadores y los recursos que pueden utilizarse, que comúnmente son tecnológicos, circunstancia que puede representar un gran obstáculo en lugares donde la tecnología no ha hecho mucho acto de presencia, como ocurre en los países de Latinoamérica. Sin embargo para el presente trabajo hablamos del juego en el sentido convencional, no en el que hace referencia la gamificación, el más importante recurso que utiliza el docente es su propia imaginación.

\section{LA ENSEÑANZA DE MATEMÁTICAS Y QUÍMICA A TRAVÉS DE JUEGO}

De entre las asignaturas tradicionalmente más complejas y difíciles para la mayoría de los estudiantes de cualquier nivel educativo, se encuentran las llamadas ciencias exactas o experimentales como las Matemáticas o la Química. En la enseñanza de este tipo de disciplinas es donde el juego toma relevancia, y se vuelve un recurso oportuno para lograr aprendizajes. Con respecto a las Matemáticas, se han instrumentado diversos juegos para facilitar la comprensión de temas como fracciones, números primos, compuestos, par, impar, punto, recta, segmento, semirrecta y ángulo, y las operaciones básicas entre otros.

Se presenta una tabla con algunos ejemplos de juegos implementados en diversos contenidos relacionados con las Matemáticas, así como la población a la que se aplicaron y su referencia (Tabla 1) para que puedan ser consultados por otros profesores interesados en estimular de manera lúdica el aprendizaje de ésta ciencia entre sus alumnos. 
Tabla 1

Juegos para enseñar contenidos de Matemáticas

\begin{tabular}{|c|c|c|c|}
\hline \multicolumn{4}{|c|}{ MATEMÁTICAS } \\
\hline Contenidos & Juegos Implementados & Población & Referencia \\
\hline Fracciones & $\begin{array}{l}\text { Fracciones rectan- } \\
\text { gulares diseñadas en } \\
\text { Ilamativos colores que } \\
\text { representan las } \\
\text { fracciones. }\end{array}$ & $\begin{array}{c}\text { Niños de sexto grado. } \\
\text { Planeta Rica-Córdoba, } \\
\text { Colombia. }\end{array}$ & $\begin{array}{c}\text { Meza, A., y Barrios, A. } \\
\text { (2010) }\end{array}$ \\
\hline $\begin{array}{l}\text { Concepto de número } \\
\text { primo, compuesto, par, } \\
\text { impar. Mayor que y } \\
\text { menor que, posición y } \\
\text { ecuaciones simples. }\end{array}$ & $\begin{array}{c}\text { Buscando el número de } \\
\text { Timoteo. Viajando por } \\
\text { la tabla. } \\
\text { Cajitas de Origami. } \\
\text { Muñecos Glotones. }\end{array}$ & $\begin{array}{l}\text { Niños de primer } \\
\text { grado. Santo Domingo, } \\
\text { República Dominicana. }\end{array}$ & Cruz, I. (2013) \\
\hline $\begin{array}{c}\text { Actividades matemáticas } \\
\text { esenciales }\end{array}$ & $\begin{array}{l}\text { Juego de rol de mesa } \\
\text { Special Agents of the } \\
\text { Universe, obra de teatro } \\
\text { sin guión }\end{array}$ & $\begin{array}{c}\text { Estudiantes de quinto } \\
\text { grado de colegio rural } \\
\text { en ciudad Bolívar, } \\
\text { Colombia. }\end{array}$ & Camargo, D. (2014) \\
\hline $\begin{array}{c}\text { Punto, recta, } \\
\text { segmento, semirrecta y } \\
\text { ángulo. }\end{array}$ & $\begin{array}{l}\text { Tabú: elementos en el } \\
\text { plano y memory } \\
\text { sexagesimal. } \\
\text { Dominó de ángulos. }\end{array}$ & $\begin{array}{l}\text { Primer grado de Escuela } \\
\text { Secundaria Obligatoria } \\
\text { (ESO). Asturias, España. }\end{array}$ & $\begin{array}{c}\text { Muñiz, L. y Rodríguez, } \\
\text { P. (2014) }\end{array}$ \\
\hline $\begin{array}{l}\text { Las cuatro } \\
\text { operaciones básicas } \\
\text { (adición, sustracción, } \\
\text { producto y cociente) }\end{array}$ & $\begin{array}{c}\text { Tangram. } \\
\text { Actividades escritas. } \\
\text { Juego diseñado. }\end{array}$ & $\begin{array}{l}\text { Estudiantes de quinto } \\
\text { grado. Quindío, } \\
\text { Colombia. }\end{array}$ & $\begin{array}{c}\text { Aristizábal, J., } \\
\text { Colorado, H. y Gutiérrez, } \\
\text { H. (2016) }\end{array}$ \\
\hline Multiplicación & $\begin{array}{l}\text { Puzzles, deportivas, } \\
\text { juegos de mesa: el } \\
\text { bingo y la oca, juegos de } \\
\text { internet, lanzamiento } \\
\text { matemático, llena la } \\
\text { cesta, los trenes, party } \\
\text { matemático. }\end{array}$ & $\begin{array}{c}\text { Alumnos de tercero } \\
\text { de primaria. Madrid, } \\
\text { España. }\end{array}$ & Rodrigo, N. (2017). \\
\hline
\end{tabular}

Fuente: elaboración propia

La Química, es también una asignatura cuya enseñanza representa un reto para los docentes, por lo que se implementan estrategias para facilitar la transferencia de sus temas entre los alumnos. Montiano (2010) reúne una serie de alternativas lúdicas para estimular el aprendizaje de la Química y el desarrollo de las competencias básicas. Franco-Mariscal et al (2012) ofrecen una gama de estrategias lúdicas para el aprendizaje de los elementos químicos. En este sentido son varias las investigaciones desarrolladas en las que se utilizaron diversos juegos para contribuir y motivar el aprendizaje de los estudiantes de una forma entretenida como se muestra a continuación (Tabla 2). 
Tabla 2

Juegos para enseñar contenidos de Química

\begin{tabular}{|c|c|c|c|}
\hline \multicolumn{4}{|c|}{ QUÍMICA } \\
\hline Contenidos & Juegos Implementados & Población & Referencia \\
\hline $\begin{array}{c}\text { Conceptos y } \\
\text { aplicación de la } \\
\text { nomenclatura de } \\
\text { Química }\end{array}$ & $\begin{array}{l}\text { Memorama, damero y sopa } \\
\text { de letras, dominó, tabla } \\
\text { periódica recitada, carrera de } \\
\text { los óxidos, modelos químicos }\end{array}$ & $\begin{array}{l}\text { Estudiantes de noveno } \\
\text { grado de educación } \\
\text { básica Sucre, Venezuela }\end{array}$ & $\begin{array}{c}\text { Valero, P. y Mayora, F., } \\
\text { (2009) }\end{array}$ \\
\hline $\begin{array}{l}\text { Diversos temas de } \\
\text { Química y Biología }\end{array}$ & $\begin{array}{c}\text { Juegos de mesa, de destreza } \\
\text { física y mental, tradicionales } \\
\text { (sopa de letras, mapas } \\
\text { mentales), recreativos, } \\
\text { videojuegos }\end{array}$ & $\begin{array}{l}\text { Estudiantes de edu- } \\
\text { cación básica y media } \\
\text { vocacional, Colombia. }\end{array}$ & $\begin{array}{c}\text { Melo, M. y Hernández, } \\
\text { R. (2014) }\end{array}$ \\
\hline $\begin{array}{l}\text { Nomenclatura de } \\
\text { química orgánica. } \\
\text { Grupos funcionales. }\end{array}$ & $\begin{array}{l}\text { Representación de estruc- } \\
\text { turas moleculares orgánicas } \\
\text { mediante unión de esferas de } \\
\text { unicel que hacen a su vez de } \\
\text { átomos y un crucigrama. }\end{array}$ & $\begin{array}{c}\text { Estudiantes de Pre- } \\
\text { paratoria Regional de } \\
\text { Atotonilco Guadalajara, } \\
\text { México. }\end{array}$ & Zaragoza, et al (2016) \\
\hline $\begin{array}{l}\text { Diversos temas de } \\
\text { Química }\end{array}$ & $\begin{array}{c}\text { Seis juegos de mesa y siete en } \\
\text { computadora, usando el pro- } \\
\text { grama JClic. Fue establecida la } \\
\text { estrategia de implementación } \\
\text { de los juegos y los escenarios } \\
\text { para su aplicación. }\end{array}$ & $\begin{array}{c}\text { Estudiantes del octavo } \\
\text { grado en Santiago de } \\
\text { Cuba. }\end{array}$ & $\begin{array}{c}\text { Plutin -Pacheco y } \\
\text { García - López (2016) }\end{array}$ \\
\hline $\begin{array}{c}\text { Diversos temas de } \\
\text { Química }\end{array}$ & $\begin{array}{c}\text { Juegos tradicionales (lotería, } \\
\text { memorama, serpientes y } \\
\text { escaleras, etc.). }\end{array}$ & $\begin{array}{l}\text { Estudiantes de nivel } \\
\text { medio superior de } \\
\text { Salvatierra, Guanajuato, } \\
\text { México. }\end{array}$ & $\begin{array}{c}\text { López, L. y Caballero, G. } \\
\text { (2017) }\end{array}$ \\
\hline
\end{tabular}

Fuente: elaboración propia

La asignatura de Química aunque es una ciencia difícil y representa un disgusto para los alumnos y un reto para los profesores, también es factible de modelarse con estrategias lúdicas que estimulen la participación de los niños y en consecuencia su aprendizaje. Los docentes comprometidos con el aprendizaje de sus estudiantes buscarán estrategias de diversa índole que les faciliten la impartición de esos temas que históricamente han sido complicados para unos y otros.

\section{DISCUSIÓN}

Furió (1994) señalaba hace más de dos décadas que el paradigma de enseñanza-aprendizaje de las ciencias (basado en la transmisión verbal del conocimiento científico) estaba acabado y aparecía en escena el paradigma emergente de orientación constructivista, pero advertía que la práctica docente estaba todavía lejos de asumir ese cambio. En este paradigma se sitúa la didáctica de las ciencias (DC) cuyo origen es situado por Ariza (1998) en los años 50 y da un recuento de su evolución como una forma de enseñar las ciencias (en especial las experimentales); además la DC es 
considerada como una disciplina práctica emergente que se incluye en el campo más amplio de las ciencias de la educación, en virtud de su interés educativo.

Una de las apuestas de la didáctica de las ciencias es la implementación del juego en la realización de las actividades de aprendizaje, lo cual representa una alternativa de innovación para los currículos experimentales. A través del juego se propicia la construcción del conocimiento y el aprendizaje significativo, tanto individual como colectivo (Torres y Torres, 2007). Este pensamiento viene a mitigar la tendencia academicista e instructivista de la tradición escolar, que impide ver el potencial lúdico en la enseñanza de materias tan complicadas como Matemáticas, ya que todavía existe la idea del juego como algo trivial y sin relevancia para uso didáctico (Camargo, 2014).

Pese al poco uso del juego como estrategia didáctica, muchos docentes aprovechan sus posibilidades como se puede ver en los ejemplos contenidos en las tablas presentadas, en los cuales se advierten muy diversas propuestas. En ellas, gran parte de los juegos diseñados e implementados tienen su base en algunos tradicionales (lotería, memorama, serpientes y escaleras). De modo que sólo hace falta adaptarlos para estimular el interés del estudiante y de ese modo, convertirlos en una herramienta que facilite la aprehensión de contenidos que en el método tradicional suele ser tan complicado.

Las experiencias referidas en los ejemplos dan cuenta de implicaciones positivas en el trabajo que se hace dentro del aula, entre las que se pueden destacar:

1. Logran la motivación de los alumnos por el estudio de las ciencias (Solbes, Lozano y García, 2008; Melo y Hernández, 2014; Plutin -Pacheco y García - López, 2016; Aristizábal, Colorado y Gutiérrez, 2016)

2. Se pueden transferir con facilidad a diversas unidades de aprendizaje (Muñiz, Alonso y Rodríguez, 2014; Zaragoza, et al, 2016)

3. Facilitan la comprensión y la significación de los temas (Valero y Mayora, 2009; Muñiz, Alonso y Rodríguez, 2014; Zaragoza, et al, 2016; Rodrigo, 2017)

4. Estimulan las capacidades de pensamiento (Melo y Hernández, 2014; Rodrigo, 2017)

5. Mejoran la producción de conocimiento científico (Melo y Hernández, 2014; López y Caballero, 2017)

6. Ayudan a establecer ambientes más favorables y disminuyen el tedio (Valero y Mayora, 2009; Cruz, 2013; López y Caballero, 2017)

7. Fomentan valores como la competitividad y el trabajo en equipo (Camargo, 2014; Aristizábal, Colorado y Gutiérrez, 2016)

Los juegos que se han descrito en las experiencias anteriores corresponden, en la clasificación de Piaget, al de tipo de reglas, en tanto se practican de una 
manera más colaborativa que individualizada, y se define por las normas asumidas por los jugadores. En la de Groos, se agrupan en los llamados de experimentación o funciones generales, en donde se involucra los sentidos, en virtud que se involucra al otro en la experiencia. En la clasificación de Gairín serían de estrategia, los cuales conllevan una serie de reclasificaciones, acordes a los que se pueden utilizar en el aula con fines didácticos. Por último, en la propuesta de Aizencang, corresponden a los de mímesis o juegos de imitación, dado que el niño emula lo que percibe del contexto de los adultos

\section{CONCLUSIONES}

Los resultados obtenidos en las experiencias expuestas, donde se aplicó el juego como recurso didáctico para la enseñanza de las ciencias, permite inferir que su efectividad no sólo está en el fin que se propone, que es el aprendizaje, sino que durante el proceso también intervienen factores que influyen en otros aspectos del sujeto como las emociones, lo cual permite elevar la motivación de quien lo realiza y tener como resultado una mayor disposición para aprender. Representa además una oportunidad para alejar de las aulas el aburrimiento, dinamizar las secuencias didácticas, facilitar la producción y transferencia del conocimiento científico entre otros beneficios. Y aunque se ha demostrado la efectividad del juego en la enseñanza-aprendizaje de diversos contenidos escolares, en asignaturas como Matemáticas y Química, no ha logrado generalizarse como un recurso o estrategia que favorezca y motive el aprendizaje de los estudiantes, ya que hasta ahora son todavía pocos los profesores que lo implementan en las aulas. Franco-Mariscal, Oliva-Martínez, y Bernal-Márquez (2012) señalan que dentro de los límites conocidos por ellos, no conocen la realización de otro estudio profundo que permita contrastar la utilidad de estos recursos (juegos) como herramientas educativas.

Por ello, es importante realizar estudios de la eficacia del juego como elemento estimulador del aprendizaje. La investigación-innovación es un binomio recurrentemente enunciado por la literatura de la didáctica de las ciencias. Por otro lado, Furió (1994) señala que tanto en la formación de futuros profesores como en el reciclaje de profesores en activo, habría que considerar la preparación de la investigación e innovación educativa como necesidad formativa de primer orden entre el profesorado de todos los niveles educativos. 


\section{REFERENCIAS BIBLIOGRÁFICAS}

Aristizábal, J. H., Colorado, H., y Gutiérrez, H. (2016). El juego como una estrategia didáctica para desarrollar el pensamiento numérico en las cuatro operaciones básicas. Sophia, 12 (1), 117-127. Recuperado de: https://bit.ly/2YMCbyF

Ariza, P. (1998). Pasado, presente y futuro de la didáctica de las ciencias. Enseñanza de las ciencias, 16 (1), 175-185.

Bernabeu N. y Goldstein A. (2008), Creatividad y Aprendizaje, el Juego como Herramienta Pedagógica, Madrid, España: Narcea.

Camargo, D. M. (2014). Juego de rol y la actividad matemática. Infancias Imágenes, 13 (2), 138-14.

Castrillón, L. T. (2017). Los juegos y su rol en el aprendizaje de una lengua. La Tercera Orilla, (19), 86-93. Recuperado de https://bit.ly/2YG5iDC

Cruz, I. M. (2013). Matemática Divertida: Una Estrategia para la enseñanza de la Matemática en la Educación Básica. I Congreso de Educación Matemática de América Central y Del Caribe. ICEмacyc. Santo Domingo, República Dominicana. Recuperado de http://ciaem-redumate.org/memoriasicemacyc/64-526-1-DR-T.pdf

Domínguez, C. T. (2015). La lúdica: una estrategia pedagógica depreciada. Colección Reportes Técnicos de Investigación, 27, 1-25. Recuperado de: https://bit.ly/2KpozF8

Foncubierta, J.M. y Rodríguez, Ch. (2014). Didáctica de la gamificación en la clase de español, recuperado en https://espanolparainmigrantes.files. wordpress.com/2016/04/didactica_gamificacion_ele.pdf

Franco-Mariscal, A.J., Oliva-Martínez, J.M. y Bernal-Márquez S. (2012). Una revisión bibliográfica sobre el papel de los juegos didácticos en el estudio de los elementos químicos. Segunda parte: los juegos al servicio de la comprensión y uso de la tabla periódica, Educación Químíca, 23(4), 474-481.

Furió, C.J. (1994). Tendencias actuales de la formación de profesorado en ciencias. Enseñanzas de las Ciencias, 12 (2) 188-199.

Gairín, J. M. (1990). Efectos de la utilización de juegos educativos en la enseñanza de las matemáticas. Educar, 17, 105-118. Recuperado de: https:// bit.ly/2weVdBf

Gallardo, J. A. (2018). Teorías sobre el juego y su importancia como recurso educativo para el desarrollo integral infantil. Revista Educativa Hekademos, (24), 41-51. Recuperado de: https://bit.ly/2QgTiFq

González, A. G., Molina, J. G., y Sánchez, M. (2014). La matemática nunca deja de ser un juego: investigaciones sobre los efectos del uso de juegos en la enseñanza de las matemáticas. Educación Matemática, 26(3), 109132. Recuperado de: https://bit.ly/2Hwsajf 
Hierro, I. M. (2014). Gamificación. El poder del juego en la gestión empresarial y la conexión con los clientes. Alicante, España. Recuperado de https:// gloriaburgos23.jimdofree.com/aprendizaje-de-lo-seres-humanos

López, L., \& Caballero, G. (2017). Química lúdica. Revista de divulgación científica, 3(2), 1753-1757. Recuperado de: https://bit.ly/2YGXRvM

Marín, V. (2015). La Gamificación educativa. Una alternativa para la enseñanza creativa, Digital Education Review (27). Recuperado en http:// greav.ub.edu/der/

Melo, M. P., y Hernández, R. (2014). El juego y sus posibilidades en la enseñanza de las ciencias naturales. Innovación Educativa, 14(66), 41-63. Recuperado de: https://bit.ly/2JxtFAo

Meza, A., y Barrios, A. (2010). Propuesta Didáctica para la Enseñanza de las Fracciones. Memoria $11^{\circ}$ Encuentro Colombiano de Matemática Educativa, 674-682. Recuperado de https://bit.ly/37dguMt

Molina, R. (2016). El concepto de juego y su importancia dentro del ámbito educativo en escolares de 10 a 12 años. Educación Física y Deportes, Revista Digital., (221), 1. Recuperado de: https://bit.ly/2Wqmsrz

Montero, B. (2017). Aplicación de juegos didácticos como metodología de enseñanza: Una Revisión de la Literatura. Pensamiento Matemático, 7(1), 75-92. Recuperado de: https://bit.ly/2LYP8DR

Montiano, M. (2010). Didáctica de la química a través de los juegos. Revista Digital Para Profesionales de La Enseñanza, (11), 1-10. Recuperado de: https://bit.ly/30CKzCg

Morales, O. R, y Urrego, Z. R. (2017). La enseñanza por medio del juego para un mejor aprendizaje. Praxis Pedagógica, (20), 123-136. Recuperado de: https://bit.ly/2CNGa77

Muñiz, L., Alonso, P., y Rodríguez, L. J. (2014). El uso de los juegos como recurso didáctico para la enseñanza y el aprendizaje de las Matemáticas: estudio de una experiencia innovadora. Revista Iberoamericana de Educación Matemática, (39), 19-33. Recuperado de: https://bit.ly/2FC4k2l

Pacheco, M. (2011). El juego en la etapa infantil. Revista Digital Para Profesionales de La Enseñanza, (17), 1-11. Recuperado de: https://bit.ly/2wbAuyf

Palacino, F. (2007). Competencias comunicativas, aprendizaje y enseñanza de las Ciencias Naturales: un enfoque lúdico, Revista Electrónica de Enseñanza de las Ciencias 6, (2), 275-298.

Plutin, N., y García, C. A. (2016). Estrategia didáctica basada en la lúdica para el aprendizaje de la química en la secundaria básica cubana. Revista Cubana de Química, 28(2), 610-624. Recuperado de: https://bit. ly/2X3tLCA

Rengel, W.A. (2018). La gamificación para la enseñanza de derecho administrativo de la carrera de derecho de la Universidad Regional Autónoma de los Andes - uniandes, tesis de Maestría en Docencia de la Universidad 
Regional Autónoma de los Andes - UNIANDES. Recuperado de https:// bit.ly/2NIoMl6

Rodrigo, N. (2017). Enseñar a multiplicar mediante el juego y el aprendizaje cooperativo, Tesis de Maestría en Educación, Madrid Universidad Internacional de La Rioja. Recuperado de: https://bit.ly/2DhXHzY

Ruiz, M. (2017). El juego: Una herramienta importante para el desarrollo integral del niño en Educación Infantil. Tesis de maestría, Universidad de Cantabria. Recuperado de: https://bit.ly/2JUCeUD

Sánchez, P. y Francesc J. (2015). Gamificación, Education in the Knowledge Society, 16 (2), 2015, 13-15.

Sánchez, S. (2016). La importancia del juego en el proceso de enseñanzaaprendizaje de una lengua extranjera. (Tesis de maestría, Palencia Universidad de Valladolid). Recuperado de https://uvadoc.uva.es/bitstream/10324/21428/1/TFG-L1446.pdf

Solbes, K., Lozano, O., y García, R. (2008). Juegos, juguetes y pequeñas experiencias tecnocientíficos en la enseñanza aprendizaje de la Física, Química y la Tecnología, Revista Investigación en la Escuela, (65), 71 -87.

Torres, C. y Torres M. (2007). El juego como estrategia de aprendizaje en el aula, Centro de Investigación para el Desarrollo Integral Sustentable Trujillo, recuperado de: https://bit.ly/2RGqffM .

Valero, P. y Mayora, F. (2009). Estrategias para el aprendizaje de la química de noveno grado apoyadas en el trabajo de grupos cooperativos, Sapiens. Revista Universitaria de Investigación, 10 (1), 109-135.

Zaragoza, E.,Orozco,L., Macías, J.,Nuñez, M,E.,Gutiérrez, R., Hernández, D. Navarro, C.,De Alba, M., Villalobos, R., Gómez, N., Cerda, R. Gutiérrez, A., Pérez, K. (2016). Estrategias didácticas en la enseñanza-aprendizaje: lúdica en el estudio de la nomenclatura química orgánica en alumnos de la Escuela Preparatoria Regional de Atotonilco, Educación Química (27), 43-51. 